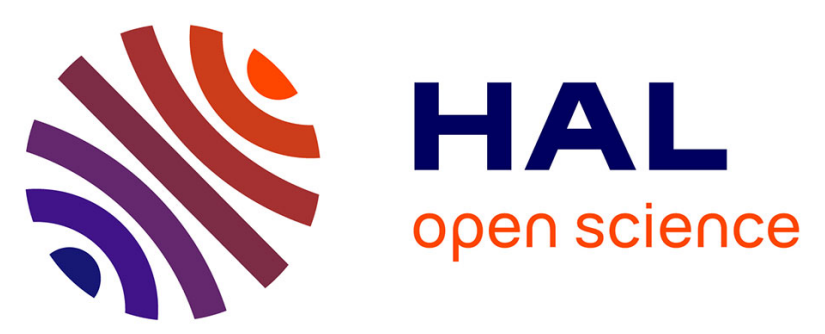

\title{
Second order approximation of the viscous Saint-Venant system and comparison with experiments
}

Theodoros Katsaounis, Chiara Simeoni

\section{To cite this version:}

Theodoros Katsaounis, Chiara Simeoni. Second order approximation of the viscous Saint-Venant system and comparison with experiments. Proceedings of the Ninth International Conference on Hyperbolic Problems, Mar 2002, CalTech, Pasadena, United States. pp.633-644, 10.1007/978-3-64255711-8_59. hal-00922694v2

\section{HAL Id: hal-00922694 \\ https://hal.science/hal-00922694v2}

Submitted on 30 Dec 2013

HAL is a multi-disciplinary open access archive for the deposit and dissemination of scientific research documents, whether they are published or not. The documents may come from teaching and research institutions in France or abroad, or from public or private research centers.
L'archive ouverte pluridisciplinaire HAL, est destinée au dépôt et à la diffusion de documents scientifiques de niveau recherche, publiés ou non, émanant des établissements d'enseignement et de recherche français ou étrangers, des laboratoires publics ou privés. 


\title{
Second order approximation of the viscous Saint-Venant system and comparison with experiments
}

\author{
Theodoros Katsaounis and Chiara Simeoni
}

Département de Mathématiques et Applications - École Normale Supérieure 45, rue d'Ulm - 75230 Paris Cedex 05 - France

katsaoun@dma.ens.fr,simeoni@dma.ens.fr

Summary. We present numerical simulations of the Saint-Venant system for shallow waters, including small friction and viscosity, motivated by the interest in recovering the results of experimental studies on the free-surface flows over an obstacle. We use the kinetic scheme "with reflections" formulated in [B. Perthame, C. Simeoni, A kinetic scheme for the Saint-Venant system with a source term, Calcolo, 38 (2001), no. 4, 201-231], appropriately extended to obtain second order accuracy according to the theory developed in [Th. Katsaounis, C. Simeoni, First and second order error estimates for the upwind source at interface method, Math. Comp. 74 (2005), no. 249, 103-122].

http://link.springer.com/chapter/10.1007\%2F978-3-642-55711-8_59

\section{Introduction}

The Saint-Venant equations for shallow waters were originally written by A. de Saint-Venant in 1871 from heuristic considerations about the mechanisms governing physical phenomena such as the flows in rivers or coastal areas (see [20]).

In a one-dimensional framework, these equations constitute simple mathematical model for the flow in ideal rectangular rivers, described at time $t \geq 0$ and at point $x \in \mathbb{R}$ through the height of water $h(t, x) \geq 0$ and its velocity $u(t, x) \in \mathbb{R}$ by means of the hyperbolic system

$$
\begin{aligned}
\frac{\partial h}{\partial t}+\frac{\partial}{\partial x}(h u) & =0, \\
\frac{\partial}{\partial t}(h u)+\frac{\partial}{\partial x}\left(h u^{2}+\frac{g}{2} h^{2}\right)+g h Z^{\prime} & =0,
\end{aligned}
$$

where $g$ denotes the gravity intensity and $Z(x)$ is the bottom topography; therefore $h+Z$ is the level of the water surface and, in what follows, we also denote the discharge by $q=h u$.

Besides, other terms can be added to the right-hand side of equation (2) to take into account further natural features of the physical context, for instance friction on the bottom and viscosity inside the fluid. 
The question to introduce more complete systems becomes crucial when one deals with the experimental verification of situations typically occurring in hydraulics, to provide a classification of different flow regimes in presence of an obstacle, for which the description based on the model (1)-(2) is unsatisfactory. Indeed, for dam breaks or hydraulic jumps, it does not allow to recover mathematically the right position with respect to the topography (see [12], for example) and the interaction of the source terms corresponding to bottom slope and friction in the shallow water equations is dominant for characterizing the steady states.

Despite its simple configuration, the shallow water flow in channels with nontrivial topography present a wide variety of regimes, producing some peculiar behaviours of the free-surface (wave trains, hydraulic jumps, turbulent profiles), which have not yet been fully examined. In [1], the results of classical analytical theories are reviewed, even though these models are validated only in the weakly nonlinear and weakly dispersive limits. Based on twodimensional numerical simulations of the nonlinear uniform potential flow around moving obstacles, an accurate description of the different types of breaking waves is proposed in [14]. However, due to the inherent limitations of the theoretical formulations, their predictions have to be interpreted in terms of experimental verifications.

We refer to [26] for a survey of the experimental study of free-surface flows over an obstacle: the behaviour of an incident subcritical channel flow is investigated for various blocking factors (namely, obstacle shape and stationary water depth); the results are analyzed in comparison with the classification schemes proposed in the previous works (see the references in that paper).

We present in this paper some numerical simulations of the Saint-Venant system, according to the experimental configuration set in [26]. To take into account dissipative effects in the physical phenomenon, we consider a modified equation for the momentum including small friction and viscosity, then the system under analysis reads

$$
\begin{aligned}
\frac{\partial h}{\partial t}+\frac{\partial}{\partial x}(h u) & =0 \\
\frac{\partial}{\partial t}(h u)+\frac{\partial}{\partial x}\left(h u^{2}+\frac{g}{2} h^{2}\right)+g h Z^{\prime} & =-\frac{g}{K^{2}} \frac{u|u|}{h^{1 / 3}}+\mu \frac{\partial}{\partial x}\left(h \frac{\partial u}{\partial x}\right),
\end{aligned}
$$

where $K$ is the Strickler's coefficient of the Manning's equation and $\mu$ denotes the kinematic viscosity of the fluid. The particular form of the source terms is suggested by empirical laws, which were originally obtained for steady state flows (refer to [25]). Similar models have been derived from the NavierStokes system for incompressible flows with a free moving boundary (see [5] and its references) and more complex laws for the friction term can also be formulated to modelize analogous problems in the case of granular media (snow avalanches, for example, as referred in [15]).

The numerical approximation of system (3)-(4) is carried out by means of the kinetic scheme proposed and extensively studied in [17]. In fact, for 
all experimental tests performed in the works quoted above, the flow was observed to be stationary and a zone of supercritical flow downstream the obstacle always occurs, though the cases examined concern the subcritical regime. So, we use a method which preserves the free-surface profile of steady states with nontrivial bottom topography and which is able to deal with transcritical regimes.

The discretization of the additional source terms in (4) is rather standard, based on a semi-implicit approach for the friction term and direct integrations by the finite volume method for the diffusive term. Moreover, when friction and viscosity are neglected, we recover the system (1)-(2). Appropriate extensions of the primitive algorithm are thus considered, in order to improve the numerical accuracy.

The paper is organized as follows. In Section 2, we recall some specific notations of the Upwind Interface Source method for hyperbolic conservation laws with geometrical source term, illustrated in [18], by extending its general formalism to the system (1)-(2). We also introduce the approximation of the dissipative terms, for treating the system (3)-(4). Connected with the numerical approach developed in [17], the question to derive a second order scheme is addressed in Section 3. We describe the experimental configuration underling our analysis in Section 4 and we present the results of numerical simulations made according to the experimental data provided in [26]. Some remarks are discussed to justify theoretical tests in comparison with the experiments.

\section{Formalism of the numerical method}

The Saint-Venant system for shallow waters (1)-(2) belongs to the class of the hyperbolic systems of balance laws, with a geometrical source term, and can be written in the equivalent form

$$
\frac{\partial \mathcal{U}}{\partial t}+\frac{\partial}{\partial x} A(\mathcal{U})=B(x, \mathcal{U})
$$

where $\mathcal{U}=(h, h u)$ represents the vector of conservative variables, the flux function is given by $A(\mathcal{U})=\left(h u, h u^{2}+\frac{g}{2} h^{2}\right)$ and $B(x, \mathcal{U})=\left(0,-g h Z^{\prime}\right)$ indicates the external term.

The equation (5) reproduces the general formalism introduced in [18] for the particular case of scalar conservation laws, so the numerical theory stated in that context formally extends to system (1)-(2), to characterize approximations with suitable theoretical properties. 


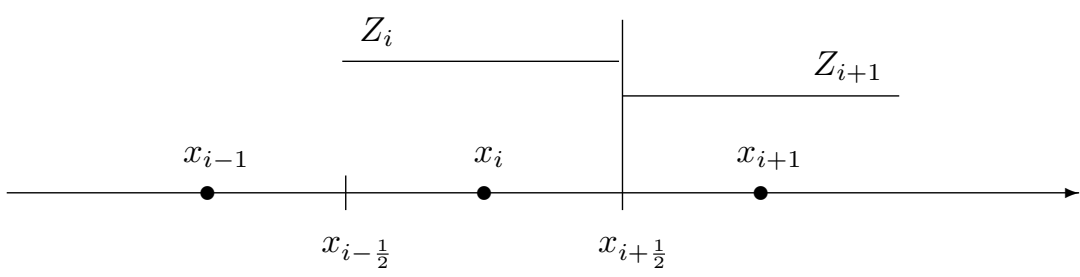

We set up a mesh on $\mathbb{R}$, whose central vertices are $x_{i}, i \in \mathbb{Z}$, made of cells $\mathcal{C}_{i}=\left[x_{i-\frac{1}{2}}, x_{i+\frac{1}{2}}\right)$ with nonuniform length $\Delta x_{i}$ and the points $x_{i+\frac{1}{2}}$ indicate the cell interfaces. We also consider a time discretization $t^{n}, n \stackrel{N}{\in}$, with variable time-step $\Delta t$. Then we construct a piecewise constant representation of the function $Z(x)$ on the mesh, with coefficients $Z_{i}=\frac{1}{\Delta x_{i}} \int_{\mathcal{C}_{i}} Z(x) d x$ for example.

A classical approach to nonlinear hyperbolic problems consists in using finite volume methods, which are designed for computations with arbitrary meshes (refer to [4], for instance). Taking into account the source term directly in the definition of the numerical fluxes, the fully explicit finite volume scheme for equation (5) is written in the compact form

$$
\mathcal{U}_{i}^{n+1}-\mathcal{U}_{i}^{n}+\frac{\Delta t}{\Delta x_{i}}\left(A_{i+\frac{1}{2}}^{n,-}-A_{i-\frac{1}{2}}^{n,+}\right)=0,
$$

where $A_{i+\frac{1}{2}}^{n, \pm}=\mathcal{A}^{ \pm}\left(\mathcal{U}_{i}^{n}, \mathcal{U}_{i+1}^{n}, \Delta Z_{i+\frac{1}{2}}\right)$, with $\Delta Z_{i+\frac{1}{2}}=Z_{i+1}-Z_{i}$, are defined by means of appropriate numerical functions $\mathcal{A}^{ \pm}=\left(\mathcal{A}_{h}^{ \pm}, \mathcal{A}_{q}^{ \pm}\right)$for each component of (5) and the following consistency properties are required,

$$
\begin{aligned}
& \mathcal{A}_{h}^{+}(\mathcal{U}, \mathcal{V}, \Delta Z)=\mathcal{A}_{h}^{-}(\mathcal{U}, \mathcal{V}, \Delta Z) \\
& \mathcal{A}_{q}^{+}(\mathcal{U}, \mathcal{V}, \Delta Z)-\mathcal{A}_{q}^{-}(\mathcal{U}, \mathcal{V}, \Delta Z)=-g h \Delta Z+\mathcal{O}(|\Delta Z|+|\mathcal{U}-\mathcal{V}|), \\
& \mathcal{A}^{+}(\mathcal{U}, \mathcal{U}, 0)=\mathcal{A}^{-}(\mathcal{U}, \mathcal{U}, 0)=A(\mathcal{U})
\end{aligned}
$$

We note that condition (7a) ensures that the numerical fluxes are conservative for the equation (1). Moreover, we have $\mathcal{A}_{i+\frac{1}{2}}^{n, \pm}=\mathcal{A}_{i+\frac{1}{2}}^{n}+\left(\mathcal{A}_{i+\frac{1}{2}}^{n, \pm}-\mathcal{A}_{i+\frac{1}{2}}^{n}\right)$ and the quantity in parentheses holds for discrete contributions of the source term at the cell interfaces, according to the Upwind Interface Source method; therefore, the relation ( $7 \mathrm{~b}$ ) guarantees consistency with the continuous model, as readily obtained by standard asymptotic expansions. We deduce from $(7 \mathrm{c})$ that the numerical scheme (6) satisfies the classical definitions for homogeneous problems.

The kinetic scheme for the Saint-Venant system proposed in [17] is compatible with the above formalism and endowed with further stability properties associated to the physical model (it preserves the steady state of still water, satisfies a discrete entropy inequality and makes non-negative water height).

In order to perform numerical simulations with experimental data, we consider the modified shallow water equations (3)-(4), for which that scheme 
applies to terms corresponding to the hyperbolic system (1)-(2).

The discretization of the friction term is implicit (see [16], for example) and splitted into two steps, only concerning the equation (4), which include the approximation of the viscous term,

$$
\begin{aligned}
& q_{i}^{n+\frac{1}{2}}-q_{i}^{n}+\frac{\Delta t}{2 \Delta x_{i}}\left(A_{q, i+\frac{1}{2}}^{n,-}-A_{q, i-\frac{1}{2}}^{n,+}\right)=\mu \frac{\Delta t}{2 \Delta x_{i}}\left(V_{i+\frac{1}{2}}^{n}-V_{i-\frac{1}{2}}^{n}\right), \\
& q_{i}^{n+1}-q_{i}^{n+\frac{1}{2}}=-\frac{\Delta t}{2} \frac{g}{K^{2}} \frac{q_{i}^{n+1}\left|q_{i}^{n+\frac{1}{2}}\right|}{\left(h_{i}^{n+1}\right)^{\frac{7}{3}}}
\end{aligned}
$$

where the numerical formulas used for calculating viscosity,

$$
V_{i+\frac{1}{2}}^{n}=\frac{h_{i}+h_{i+1}}{2} \frac{u_{i+1}-u_{i}}{\Delta x_{i+\frac{1}{2}}},
$$

with $\Delta x_{i+\frac{1}{2}}=\frac{\Delta x_{i}}{2}+\frac{\Delta x_{i+1}}{2}$, are derived by means of simple finite volume integrations on the mesh cells and appropriate approximations of the resulting interfacial values (we note that the discretization (10) can be reinterpreted according to the classical first order finite element method). Some different methods for processing friction terms are proposed in [3], [6] and [7].

\section{Second order schemes}

To obtain second order extensions of finite volume schemes in form (6), a rather geometrical approach is based on slope limiter techniques.

We construct a piecewise linear approximation of the function $Z(x)$ on the mesh, whose coefficients are $Z_{i}+\left(x-x_{i}\right) Z_{i}^{\prime}, x \in \mathcal{C}_{i}$, and we denote by $Z_{i}^{\prime}$ the numerical derivatives computed by applying an appropriate slope limiter (we refer to [8] and [13] for a survey of these discrete operators).

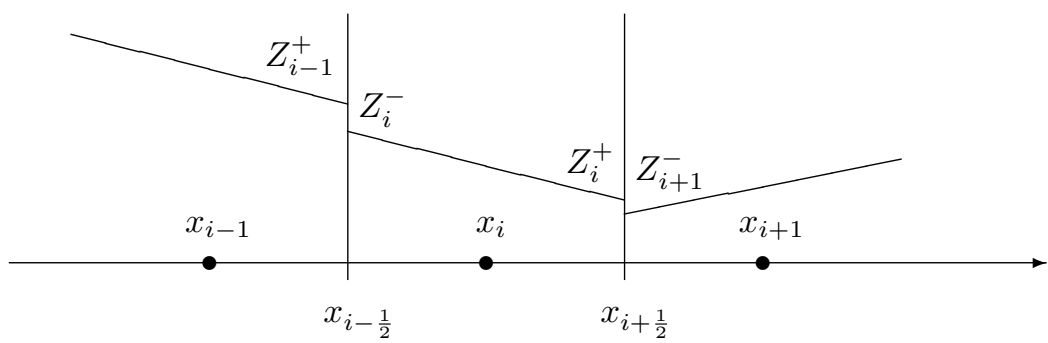

According to the arguments in [11], a second order scheme for the Interfacial Source method formally reads

$$
\mathcal{U}_{i}^{n+1}-\mathcal{U}_{i}^{n}+\frac{\Delta t}{\Delta x_{i}}\left(A_{i+\frac{1}{2}}^{n,-}-A_{i-\frac{1}{2}}^{n,+}\right)+B_{i}^{n}=0,
$$


where the numerical fluxes $A_{i+\frac{1}{2}}^{n, \pm}=\mathcal{A}^{ \pm}\left(\mathcal{U}_{i}^{n,+}, \mathcal{U}_{i+1}^{n,-}, \Delta Z_{i+\frac{1}{2}}\right)$ use the interfacial values of piecewise linear reconstructions of the numerical functions,

$$
\mathcal{U}_{i}^{n, \pm}=\mathcal{U}_{i}^{n} \pm \frac{\Delta x_{i}}{2} \mathcal{U}_{i}^{n \prime}, \quad Z_{i}^{ \pm}=Z_{i} \pm \frac{\Delta x_{i}}{2} Z_{i}^{\prime}
$$

and therefore $\Delta Z_{i+\frac{1}{2}}=Z_{i+1}^{-}-Z_{i}^{+}$in this case.

The additional discrete source term is defined by $B_{i}^{n}=\left(0, g Z_{i}^{\prime} h_{i}^{n}\right)$. Although other methods exhibit noticeable improvements, it was shown in [11] that the centered term $B_{i}^{n}$ is necessary to achieve second order accuracy for the Upwind Interface Source method, if the slope limiter used to construct the values (12) is correctly defined (see [21], [22] and [23]).

For the sake of simplicity, we consider in (11) only the first order discretization in time. It is standard to obtain higher order accuracy by applying Runge-Kutta methods for instance (see [9], [10] and its references).

The second order scheme (11) is validated by the numerical results obtained for the steady states of the Saint-Venant system (1)-(2).

\section{Experimental configuration and numerical results}

The situation studied in [26] is the one-dimensional flow of an incompressible fluid over an obstacle on the bottom of a smooth rectangular channel (see Figure 1).

The parameters of the problem are the stationary water depth $H$ and the mean velocity $U$, the kinematic viscosity of the fluid $\mu$, the characteristic length of the obstacle $L$ and its height $Z_{0}$. By combining these values, we define some specific dimensionless numbers: the Froude number $F_{0}=\frac{U}{\sqrt{g H}}$, which relates the depth-averaged flow velocity to the characteristic wave propagation speed, in the long waves approximation; the blocking factor $\alpha=\frac{Z_{0}}{H}$, controlling the flow linearity in the absence of other perturbations (when $\alpha$ tends to zero, the flow becomes linear); the obstacle ratio $\beta=\frac{Z_{0}}{L}$, which can be interpreted as a control on the flow hydrostaticity (by analogy with the long waves approximation); the Reynolds number $R e=\frac{H U}{\mu}$, for the simple case of an ideal rectangular channel.

We note that the definitions above correspond to simplifications adapted to the theoretical system described by (3)-(4), when the fluid density and the channel width are formally reduced.

The experiments have been carried out in a channel of length $12 \mathrm{~m}$, inclined at slope 0.002 and entirely made of glass. Two obstacle shapes are considered, a smooth Gaussian bump given by $Z(x)=Z_{0} \exp \left(-\frac{x^{2}}{2 L^{2}}\right)$, with ratio $\beta=0.23$, and a semi-circular bump with ratio $\beta=0.58$ (this last shape is commonly used for experimental analyses). Two sizes $Z_{0}=1.7 \mathrm{~cm}$ and $Z_{0}=4.1 \mathrm{~cm}$ are fixed for each shape, which allow to access a wider range of $\alpha$ values $(0.147 \leq \alpha \leq 0.7)$. The tests are performed in subcritical regime 


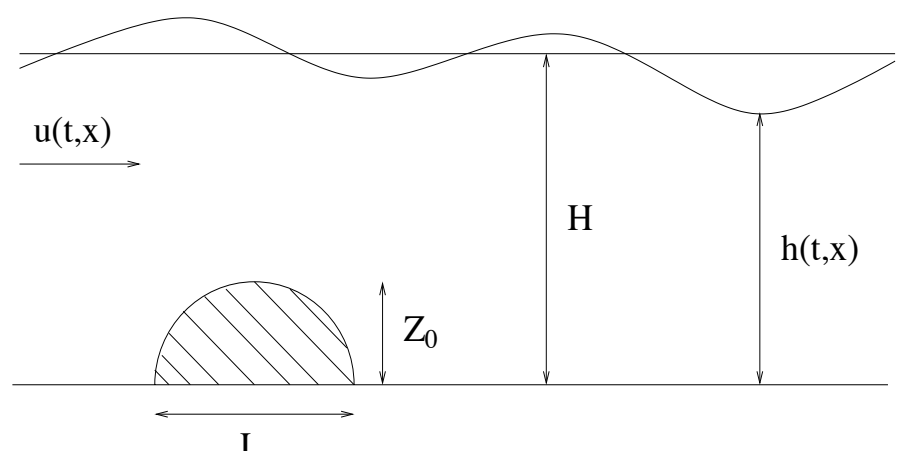

$\mathrm{L}$

Fig. 1. Experimental configuration

$\left(F_{0}<1\right)$ and the obstacles are placed in the channel so that fully developed turbulent flow conditions are attained before the obstacle.

Table 1.

\begin{tabular}{|c|c|c|c|c|}
\hline$F_{0}$ & $Z_{0}$ & $\alpha$ & $\beta$ & $R e$ \\
\hline 0.62 & $1.7 \mathrm{~cm}$ & 0.179 & 0.58 & $3.08 * 10^{4}$ \\
\hline 0.66 & $1.7 \mathrm{~cm}$ & 0.26 & 0.58 & $1.51 * 10^{4}$ \\
\hline 0.64 & $4.1 \mathrm{~cm}$ & 0.68 & 0.58 & $1.32 * 10^{4}$ \\
\hline
\end{tabular}

We present some numerical results corresponding to the test cases illustrated in Table 1, for which experimental data are available to make direct comparisons. The pairs of figures reproduce the level of the water surface and the local Froude number in the vicinity of the obstacle, obtained at time $T=150 \mathrm{~s}$ (when the flow has become completely stationary), normalized with respect to the obstacle size. According to the experiments, the flow is classified by means of the free-surface profile and three different regimes are observed as function of the blocking factor. The boundaries of each regime, in terms of $\alpha$ values, are essentially independent of the obstacle shape.

Several tests have been performed also varying the Strickler's coefficient $K$ introduced in (4), to evaluate the physical adequacy of the friction law (refer to [19] for a more precise discussion). The nonuniform mesh used for the numerical simulations is refined around the obstacle or in regions of stiff topographical variations, with a minimal mesh size of $5 * 10^{-4} \mathrm{~m}$.

The subcritical flow downstream of the obstacle displays many of the features of a supercritical flow behind a sluice gate with ensuing hydraulic jumps, implying a certain independence of the upstream conditions. The treatment of the boundary conditions, which turns out to be crucial for the numerical accuracy, is provided by the method developed in [2]. 


\section{Regime I $(\alpha \leq 0.25)$}

The graphics of the local Froude number show a transition from supercritical to subcritical flow downstream of the obstacle, through a hydraulic jump (Figure 3); the flow remains subcritical downstream the jump. The lower boundary of this regime is expected to be the value of $\alpha$ below which a classical subcritical regime should occur.

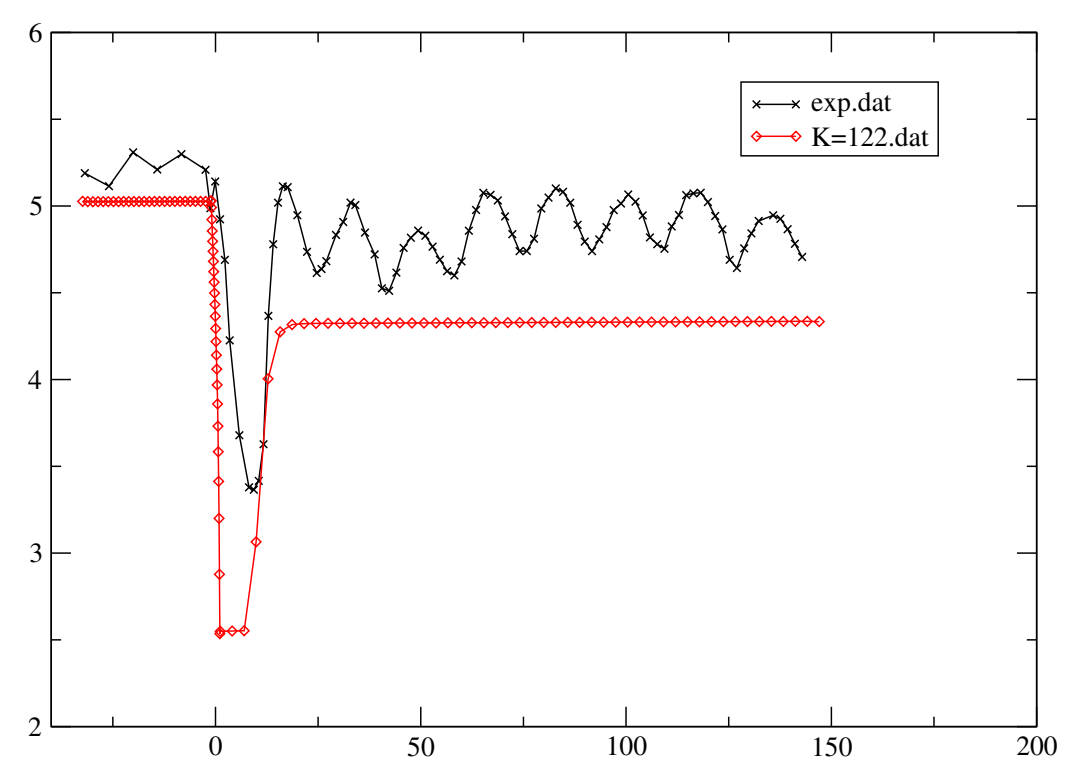

Fig. 2. Water surface $(\alpha=0.179)$

The results of the experimental test reveal the presence of a wave train on the free-surface profile (Figure 2). Moreover, some photographs taken during the experiments show bubbles arising on the crest of the hydraulic jump, as consequence of the turbulent motion. These phenomena are not taken into account in the mathematical model (3)-(4) and cannot be obtained numerically (we refer to [24] for further analysis).

\section{Regime II $(0.26 \leq \alpha<0.68)$}

The graph of the local Froude number shows that a region of supercritical flow appears downstream the obstacle, followed by a transition from supercritical to subcritical flow through a hydraulic jump (Figure 5); however, in this regime, the local Froude number undergoes further transitions and its values remain close to $F=1$ downstream the jump.

In the experimental tests, the local Froude number is oscillating around the critical value and the wave train on the free-surface profile becomes a 


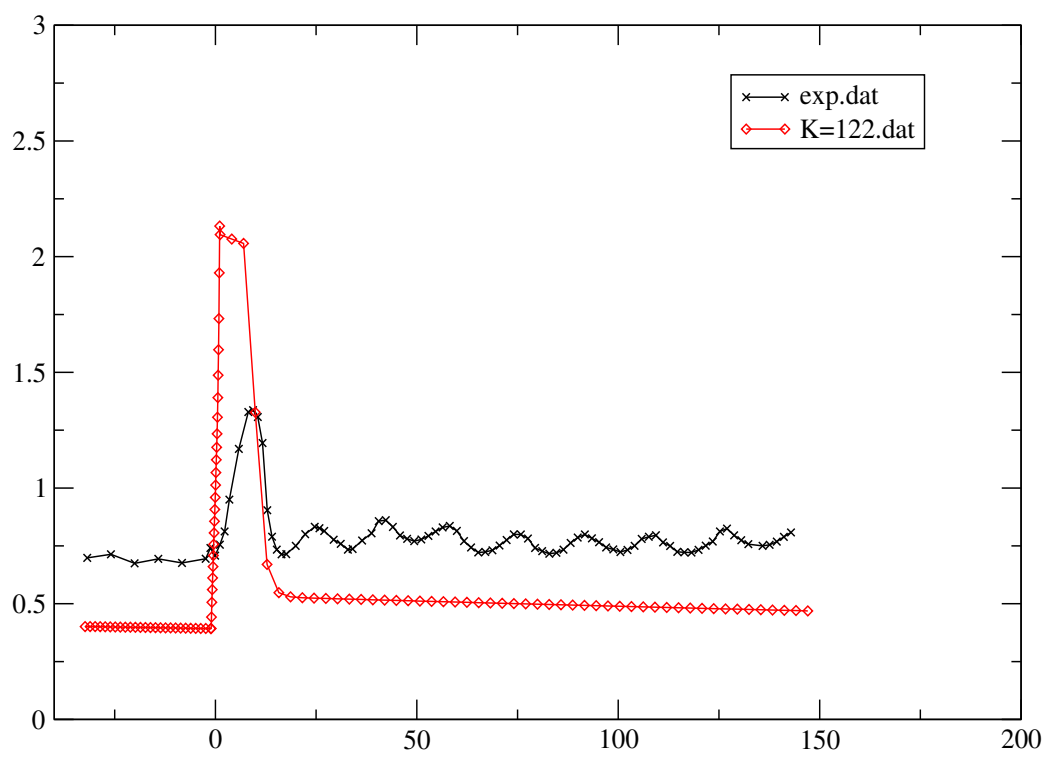

Fig. 3. Local Foude number

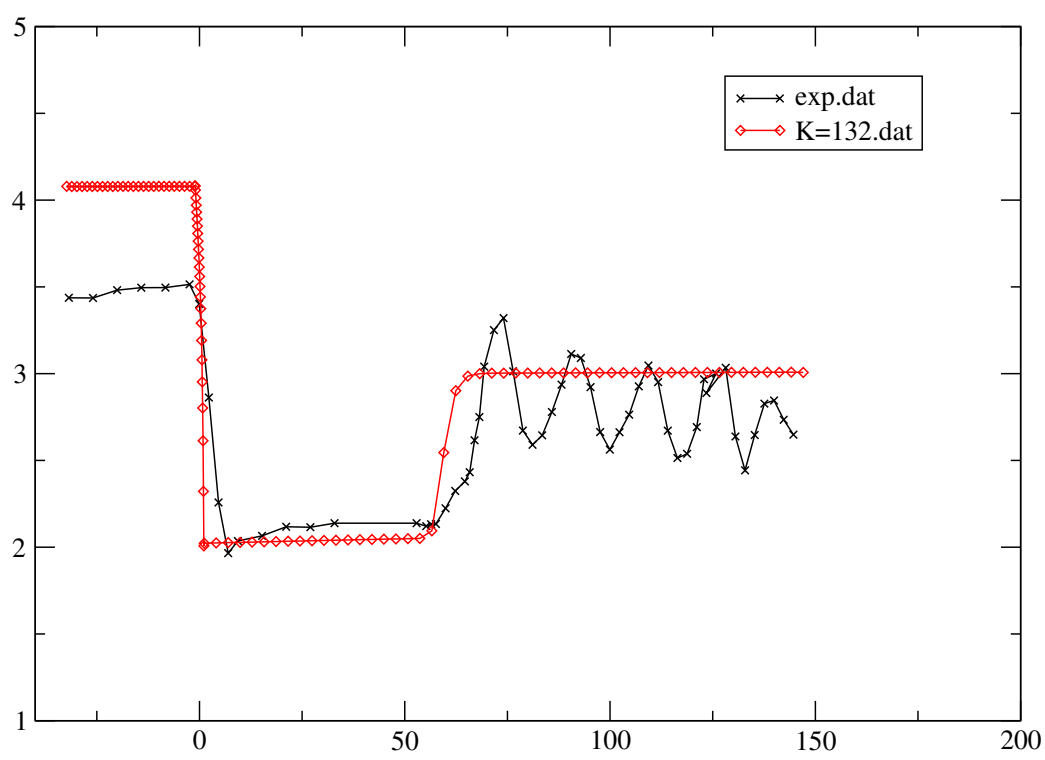

Fig. 4. Water surface $(\alpha=0.26)$ 


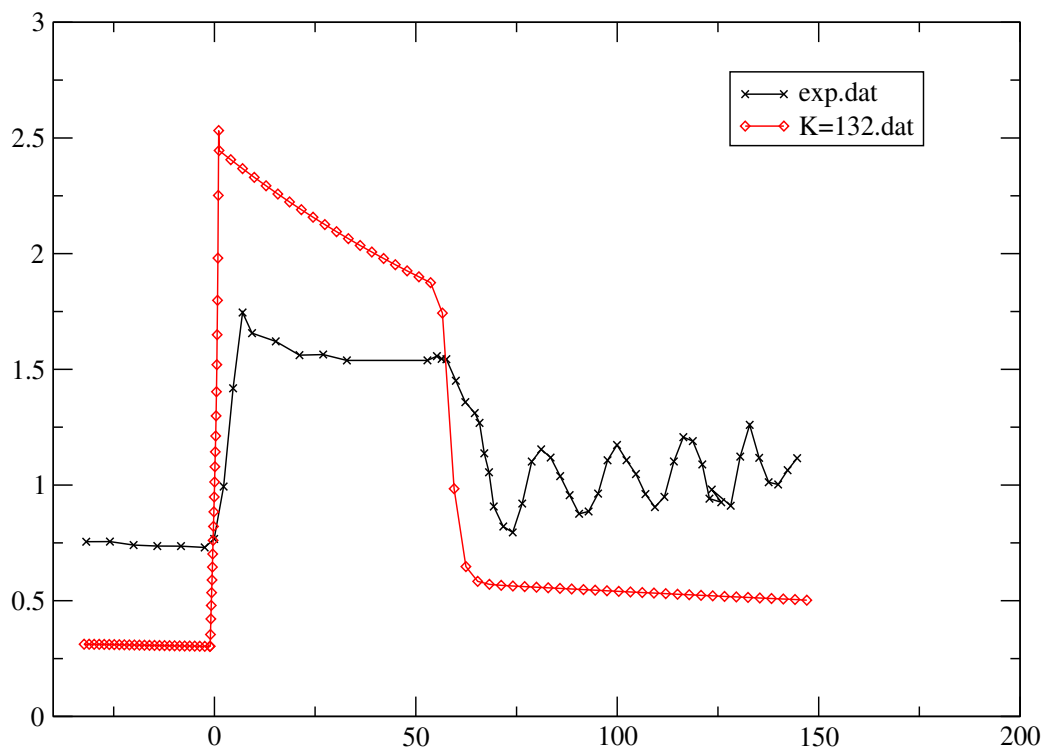

Fig. 5. Local Foude number

series of triangular hydraulic jumps. This flow regime is essentially threedimensional, the friction on the channel walls plays a significant role for the energy dissipation across the jumps (inducing water deceleration) and confinement effects seem to control the behaviour of the flow. Theoretical values obtained for the water depth (Figure 4) are higher than the experimental ones, suggesting that the numerical results are necessarily inaccurate.

Nevertheless, as two-dimensional side wall effects have not been considered in the model (3)-(4), it seems impossible to reproduce all the phenomena with one-dimensional simulations.

\section{Regime III $(\alpha \geq 0.68)$}

The experiments performed until $\alpha=0.7$ show that the flow remains supercritical downstream the obstacle (Figure 7); in other words, no hydraulic jumps arise in this regime. Small perturbations appear on the free-surface profile, in form of a wave train located downstream the obstacle (Figure 6).

We remark a rather good agreement with the experimental results concerning the position of hydraulic jumps and the length of the supercritical region predicted by the shallow water equations (3)-(4), when friction and viscosity are included. 


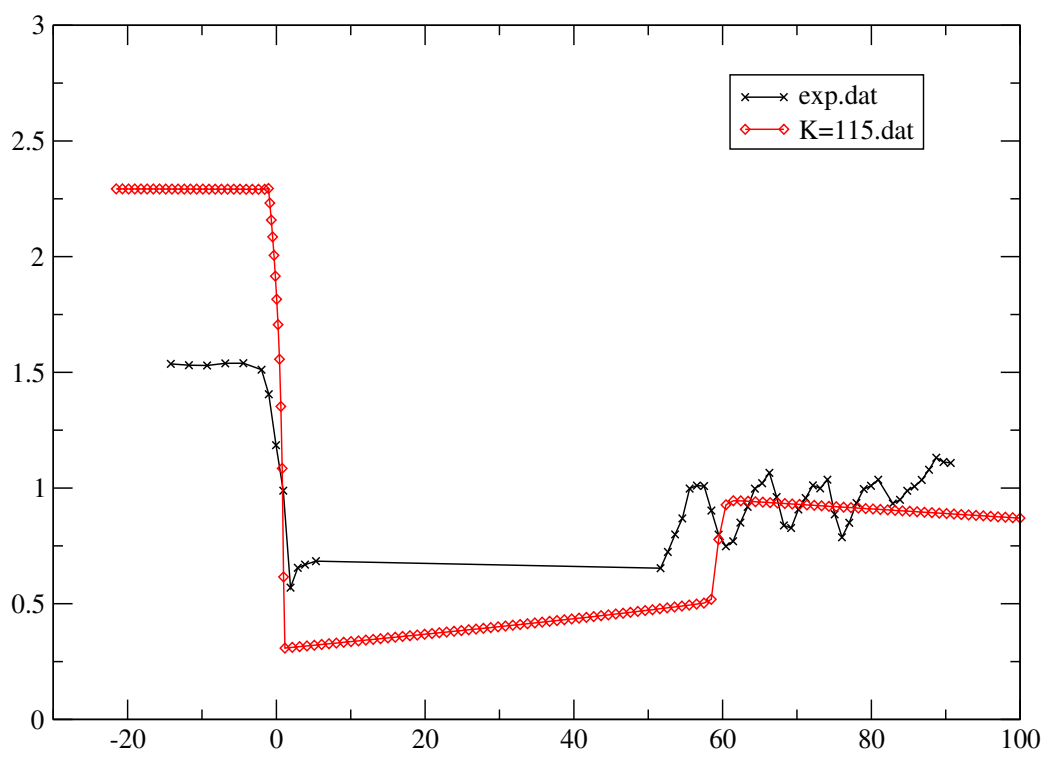

Fig. 6. Water surface $(\alpha=0.68)$

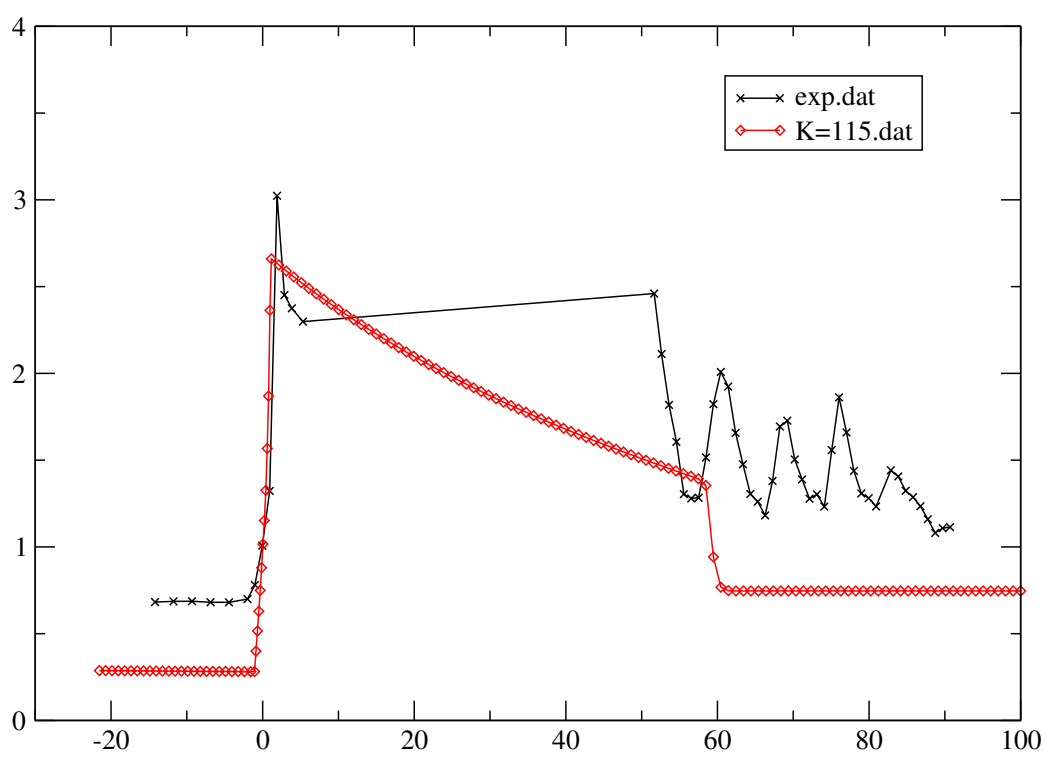

Fig. 7. Local Foude number 


\section{Acknowledgments}

This work was partially supported by the ACI "Modélisation de processus hydrauliques à surface libre en présence de singularités" (Ministre de la Recherche - France).

We would like to thank François Bouchut (DMA-ENS) and Marie-Odile Bristeau (INRIA) for several helpful discussions.

\section{References}

1. P.G. Baines, Topographic effects in stratified flows, Cambridge Monographs on Mechanics, Cambridge University Press, Cambridge, 1995

2. M.O. Bristeau, B. Coussin, Boundary conditions for the shallow water equations solved by kinetic schemes, INRIA Report, 4282 (2001)

3. A. Chinnayya, A.Y. Le Roux, A new general Riemann solver for the shallow water equations with friction and topography, preprint

4. R. Eymard, T. Gallouët, R. Herbin, Finite Volume Methods, Handbook of numerical analysis, vol. VIII, P.G.Ciarlet and J.L.Lions editors, Amsterdam, North-Holland, 2000

5. J.F. Gerbeau, B. Perthame, Derivation of viscous Saint-Venant system for laminar shallow water; numerical validation, Discrete Contin. Dyn. Syst. Ser.B, 1 (2001), no. 1, 89-102

6. P. Glaister, The efficient prediction of shallow water flows. I. Theory, Comput. Math. Appl., 32 (1996), no. 12, 129-143

7. P. Glaister, The efficient prediction of shallow water flows. II. Application, Comput. Math. Appl., 33 (1997), no. 9, 115-141

8. E. Godlewski, P.A. Raviart, Hyperbolic systems of conservation laws, Mathmatiques \& Applications, no. 3/4, Ellipses, Paris, 1991

9. S. Gottlieb, C.W. Shu, Total variation diminishing Runge-Kutta schemes, Math. Comp., 67 (1998), no. 221, 73-85

10. S. Gottlieb, C.W. Shu, E. Tadmor, Strong stability-preserving high-order time discretization methods, SIAM Rev., 43 (2001), no. 1, 89-112

11. Th. Katsaounis, C. Simeoni, First and second order error estimates for the upwind source at interface method, Math. Comp. 74 (2005), no. 249, 103-122

12. G.A. Lawrence, Steady flow over an obstacle, J. Hydraul. Engng, ASCE, 113 (1987), no. 8, 981-991

13. R.J. LeVeque, Numerical methods for conservation laws, Lectures in Mathematics ETH Zürich, Birkhäuser Verlag, Basel, 1990

14. K. Lowery, S. Liapis, Free-surface flow over a semi-circular obstruction, Internat. J. Numer. Methods Fluids, 30 (1999), 43-63

15. A. Mangeney-Castelnau, J.P. Vilotte, M.O. Bristeau, B. Perthame, C. Simeoni, S. Yernini, Numerical modelling of avalanches based on Saint-Venant equations using kinetic schemes, submitted to Journal of Geophysical Research

16. A. Paquier, Modélisation et simulation de la propagation de l'onde de rupture de barrage, PhD Thesis, Université J. Monnet, St-Étienne (France), 1995

17. B. Perthame, C. Simeoni, A kinetic scheme for the Saint-Venant system with a source term, Calcolo, 38 (2001), no. 4, 201-231 
18. B. Perthame, C. Simeoni, Convergence of the Upwind Interface Source method for hyperbolic conservation laws, preprint

19. Pukhnacheva, T.P., The problem of determining the roughness coefficient for a flow in an open channel, Prikl. Mekh. Tekhn. Fiz., 38 (1997), no. 3, 93-98; translation in J. Appl. Mech. Tech. Phys., 38 (1997), no. 3, 412-416

20. A. J.C. de Saint-Venant, Théorie du mouvement non-permanent des eaux, avec application aux crues des rivières at à l'introduction des marées dans leur lit, C.R. Acad. Sci. Paris, 73 (1871), 147-154

21. C.W. Shu, S. Osher, Efficient implementation of essentially nonoscillatory shock-capturing schemes, J. Comput. Phys., 77 (1988), no. 2, 439-471

22. C.W. Shu, S. Osher, Efficient implementation of essentially nonoscillatory shock-capturing schemes II, J. Comput. Phys., 83 (1989), no. 1, 32-78

23. C.W. Shu, High order ENO and WENO schemes for computational fluid dynamics. High-order methods for computational physics, pp. 439-582, Lect. Notes Comput. Sci. Eng., 9, Springer, Berlin, 1999

24. S. Soares-Frazão, Y. Zech, Undular bores and secondary waves. Experiments and hybrid finite-volume modelling, Journal of Hydraulic Research, 2002

25. P.L. Viollet, J.P. Chabard, P. Esposito, D. Laurance, Mécanique des fluides appliquée, Presses des Ponts et Chaussées, Paris, 1998

26. F. Vigie, O. Eiff, D. Astruc, Experimental study of the flow regimes of subcritical channel flow over an obstacle, submitted to Riverflow 2002 